\title{
Fetal Weight Nomogram for Nepalese Population
}

\section{Joshi BR}

\author{
Dept. of Radiology, Tribhuvan University Teaching Hospital
}

\begin{abstract}
Introduction: Determination of fetal weight is important in all pregnancies. Accurate antenatal assessment of the fetal weight is essential for deciding the plan of management that will minimize the perinatal morbidity and mortality rate.
\end{abstract}

Methods: This prospective longitudinal study was based on 221 low-risk pregnancies. Gestational age was computed from last menstrual period (LMP). Biparietal diameter (BPD), head circumference (HC), abdominal circumference (AC) and femoral length (FL) were measured using ultrasound and Estimated fetal weight (EFW) was calculated.

Results: Intrauterine growth expressed by EFW showed a continuous pattern until term.

Conclusion: The presented growth chart is recommended as robust reference ranges for assessing growth.

Keywords: Estimated fetal weight, nomogram, ultrasound fetal biometry.

\section{INTRODUCTION}

Assessment of fetal growth is an essential part of the fetal evaluation during pregnancy. Campbell introduced the measurement of fetal abdominal circumference (AC) to assess fetal weight in $1975 .{ }^{1}$ Many factors and measurements have been identified since then.

The objective of the present study was to establish reference ranges for the growth of estimated fetal weight (EFW) between the gestational ages of 25-41 weeks for Nepalese population.

Correspondence to: Dr. B.R. Joshi

Department of Radiology,

TUTH, Kathmandu, Nepal

Email: bjoshi01@yahoo.com

\section{METHODS}

This prospective longitudinal study was based on 221 low-risk pregnancies. The women, who visited the author's hospital from January to June 2009, participated voluntarily in the study and gave their written consent. Gestational age was computed from last menstrual period (LMP). Biparietal diameter (BPD), head circumference (HC), abdominal circumference (AC) and femoral length (FL) were measured using ultrasound and EFW was calculated using the formula of Hadlock. ${ }^{2}$ The statistical analysis was based on regression analysis.

The criteria for BPD used were that the transducer must be positioned at the correct cephalo-caudal position so that the plane 
of section intersects the third ventricle and thalamus; the transducer must be perpendicular to the parietal bones so that the margin of the calvaria is symmetrical and outer to the inner distance is measured.

The criteria for $\mathrm{HC}$ used were that the correct plane of section is through the third ventricle and thalamus in the central position of the brain showing cavum septi pellucidi in the anterior portion of the brain giving rise to 'arrow' appearance ${ }^{3}$, calvaria must be smooth and symmetrical bilaterally and the cursors should be positioned at the outer edge.

The criteria for FL used were that the transducer must be aligned to the long axis of the diaphysis and the cursors are positioned at the junctions of the bone with cartilage.

The criteria for $\mathrm{AC}$ used were that the correct cephalo-caudal plane is position where the right and left portal veins are continuous with one another depicting the shortest length of the umbilical segment of the left portal vein giving rise to 'hockey stick' appearance, the appearance of the lower ribs is symmetrical and the ellipse is fit to the skin edge.

The inclusion criteria were history of amenorrhoea, certain date of the first day of the last normal menstrual period and regular menstrual cycles ( 26 - 30 days) prior to pregnancy. The exclusion criteria were multiple gestations, uncertain first day of last normal menstrual period, irregular menstrual cycle inrtrauterine growth retardation (IUGR), menstrual cycles less than 26 or greater than 30 days, major fetal abnormalities and maternal complications - hypertension, pre-eclampsia, abnormal glucose tolerance test, diabetes mellitus, abruptio placentae, isoimmunisation.

Informed consent was received from the patients. Consent was also received from the concerned institutional review board.

\section{RESULTS}

This was a prospective study in which obstetric ultrasound was performed on 221 women and various fetal biometric parameters namely BPD, HC, AC, and FL were recorded. The subjects ranged from 16 to 38 years with a mean of 26 years. Correlation and regression analysis was done to derive the formula for the estimation of fetal weight. (Table 1)

Intrauterine growth expressed by EFW showed a continuous pattern until term. (Table 2)

Table 1: Regression equations derived from study for predicting fetal weight from $G A$.

\begin{tabular}{|c|l|c|}
\hline Parameter & \multicolumn{1}{|c|}{ Regression Equation } & $\mathbf{R}^{\mathbf{2}} \mathbf{( \% )}$ \\
\hline FW & $-2272+130.59 \mathrm{GA}$ & 84 \\
\hline FW & $-59.66-34.58+2.85 \mathrm{GA} 2$ & 86 \\
\hline FW & $3614.53-459.8 \mathrm{GA}+18.34 \mathrm{GA} 2-0.18 \mathrm{GA} 3$ & 87 \\
\hline GA & $19.354+0.006 \mathrm{FW}$ (grams) & \\
\hline
\end{tabular}


Table 2: Estimated fetal weight values for Nepalese population.

\begin{tabular}{|c|c|}
\hline GA & FW (grams) \\
\hline 25 & 950 \\
\hline 26 & 1150 \\
\hline 27 & 1350 \\
\hline 28 & 1450 \\
\hline 29 & 1650 \\
\hline 30 & 1850 \\
\hline 31 & 2050 \\
\hline 32 & 2150 \\
\hline 33 & 2350 \\
\hline 34 & 2450 \\
\hline 35 & 2650 \\
\hline 36 & 2890 \\
\hline 37 & 2950 \\
\hline 38 & 3150 \\
\hline 39 & 3350 \\
\hline 40 & 3450 \\
\hline 41 & 3650 \\
\hline & \\
\hline
\end{tabular}

\section{DISCUSSION}

Fetal growth is exponential after an initial period of about five weeks. Embryonic and fetal weight gain during the first half of pregnancy is slow, but beyond 20 weeks' gestation, there is rapid weight gain until a plateau is reached at 38 weeks. $^{4}$ Fetal size in the second trimester is a determinant of birth weight and pregnancy duration, small fetuses having lower birth weights and longer pregnancies. A concept of individually assigned pregnancy duration according to growth rates should be considered rather than a standard of 280-282 days on all pregnancies. ${ }^{5}$

Fetal weight in the normal pregnancy is considered to have a curvilinear relationship with gestational age; however, the rate of cell mitosis is less in early pregnancy, and thus, the fetus gains approximately $95 \%$ of its weight after the $20^{\text {th }}$ week of gestation. ${ }^{6}$ At term a relative reduction in both space and nutrients influences a reduction in the rate of growth. The estimates of fetal weight by means of predicted normal ultrasonic weight performed by Hadlock are more likely to be lower than actual neonatal weight. ${ }^{7}$

Chung, J.H. et al concluded that at least some of the differences in low birth weight that are noted among ethnic minorities may be attributable to ethnic differences in birth weight by GA. Their multiple linear regression models support the findings that GA is the most important predictor of birth weight and that log birth weight increases with GA in a quadratic fashion. ${ }^{8}$

However, Dawka K and Das GC have stated that ultrasonographic fetal biometry has been assumed to be more accurate than clinical methods for the estimation of fetal weight. This is largely because the presumption has been that the sonographic measurement of multiple linear and planar dimensions of the fetus provides sufficient parametric information to allow for accurate algorithmic reconstruction of the three-dimensional fetal volume of varying tissue density. ${ }^{9}$

Campbell, Wilkin and Higginbottom et al. have demonstrated that $\mathrm{AC}$ is a good index of weight in the fetus. Regression models were based on measurements of AC, HC, BPD, and FL, both alone and in combination. The best results $(1 \mathrm{SD}=7.5 \%$ of actual weight $)$ were obtained by combining measurements of fetal head, abdomen and femur, most likely due to the strong linear relationship between femur length and crown-heel length. ${ }^{10}$

Sonographic parameters in an attempt to estimate fetal weight were - BPD as an index of head size, AC as an index of body size and FL as an index of crown heel length. ${ }^{11}$

Measurements of the must be made with 
obsessional precision. Meticulous attention should be paid to the fetal anatomy. The accuracy for dating a pregnancy at 18-20 week GA is plus $\backslash$ minus one week. ${ }^{12,13}$

Perception of the fetal femur in the far field as curved is common in sonographic studies. The mild concavity of the medial aspect of the femoral diaphysis accounts for this apparent curvature, which should not be mistaken for fetal abnormality. The femur is measured from the proximal diaphysis to the distal metaphysis. The ossified portion of the femoral shaft is intensely echogenic and results in posterior shadowing. The femur nearest to the transducer appears straight. The apparent curvature of the fetal femur farthest from the transducer is a normal aberration of ultrasound imaging that should not be mistaken for fetal abnormality. ${ }^{14}$

Chatterjee, M.S. et al performed a study regarding the relationship of fetal foot length and GA. He concluded that there was statistically significant and linear correlation between fetal foot length and GA with < $0.0001 .{ }^{15}$

The study done by Johnson et al showed there was some effect of maternal and fetal factors on EFW. ${ }^{16}$ Gardosi et al reported that gestation, gender, maternal height, and smoking influenced birthnweight. ${ }^{17,18}$ That also found that maternal weight at first visit, ethnic group, and parity were significant determinants of birth weight in the population. Chauhan et al reported that the error with most EFW - models is significantly higher for fetuses in breech than in vertex position. ${ }^{19}$

\section{CONCLUSION}

Since maternal height and ethnicity influence the fetal weight, the presented growth chart is recommended as robust reference ranges for assessing growth in Nepalese population.

\section{ACKNOWLEDGEMENTS}

The author thanks all the subjects as well as the assistants who helped in the process of this research.

\section{REFERENCES}

1. Campbell S, Wilkin D . Ultrasonic measurement of fetal abdomen circumference in the estimation of fetal weight. $\mathrm{Br} J$ Obstet Gynaecol 1975;82:689-697.

2. Hadlock FP, Harrist RD, Mortinez- Poyer J. Ii utero analysis of fetal growth:a sonographic weight standard. Radiology 1991;181:129-133.

3. Filly RA and Hadlock FP. Sonographic determination of menstrual age. In: Callen PS,ed. Ultrasonography in Obstetrics \& Gynecology. $4^{\text {th }}$ Edition. New York: W B Saunders Company,2002;154-156.

4. Vorher A. Factors influencing fetal growth. Am. J.Obstet.Gynecol 1982.142:577

5. Johnsen SL, Wilsguard. Rasmussen S, Hanson MA, Godfry KM, Kiserud T. Fetal size in the second trimester is associated with the duration of pregnancy, small fetuses having longer pregnancies. BMC Pregnancy and Childbirth 2008,8:25.

6. Gruenwald,P Growth of human fetus 1 . Normal growth and its variation. Am J Obstet Gynecol 94:3102, 1966.

7. Hanarvor,M, Allahyari M, Dehbashi S. A simple estimated fetal weight equation for fetuses between 24 and 34 weeks of gestation. Int J Gynecol Obstet 1999; 67:67. 
8. Chung JH, Boscardin WJ, Garite TJ, Lagrew DC, Porto M. Ethnic differences in birth weight by gestational age: At least a partial explanation for the hispanic epidemiological paradox? Am J Obstet Gynecol 2003;189:1058-1062.

9. Dawka K and Das GC. Estimation of fetal birth weight using ultrasonographic parameters. JNMA J Nepal Med Assoc 2000;39:284-286.

10. Campbell S. Gestational age determination; second trimester. In: Chernovate FA, Isacson, GC and Campbell S Eds. Ultrasonography in Obs and Gyn.1st ed.Boston:Little Brown, 1993;303-309.

11. Nahum GG and Stainislaw $H$. Ultrasonographic prediction of term birth weight: How accurate is it? Am J Obstet Gynecol 2003;188:566-574.

12. Hadlock FP, Harrist RB, Carpenter RJ, Deter RC, Park SK. Sonographic estimation of fetal weight. Radiology 1984; 150:535-540.

13. Spinillo E, Capuzzo E, Piazi G, Baltaro F, Stronati A. Significance of low birth weight for gestational age among very preterm infants. $\mathrm{Br} J$ of $\mathrm{Obs} \& \mathrm{Gyn}$ 1997;104:668-673.

14. Abrams SL and Filly RA. Curvature of the fetal femur: a normal sonographic finding. Radiology 1985; 156:490.

15. Chatterjee MS et al. Fetal foot: evaluation of gestational age. 1994. www.the fetus. Net.

16. Johnson SL. Rasmussen S, Wilsgaard T, Sollein R, Kiserud T. Longitudinal reference ranges for estimated fetal weight . Acta obstricia et gynecologica
$2006 ; 85: 286-297$.

17. Gardosi J, Chang A, Kalian B, Sahota D, Symonds EM. Customized antenatal growth charts. Lancet 1992;339:283287.

18. Gardosi J, Mongolli M, Wilcox M, Chang A. An adjustable Fetal weight standard. Ultrasound obstet gynecol 1995;6: 168174.

19. Chauhan SP,Magann EF, Naef RW, Martin JN, Morrison JC. Sonographic assessment of birth weight among breech presentations. Ultrasound obstet gynecol 1995;6:54-57. 